\title{
DISEÑO DE UN CONTROLADOR LQG PARA UN HELICOPTERO DE TRES GRADOS DE LIBERTAD
}

\author{
Hernando González Acevedo 1, Yosman Alexis Guerrero Gómez ${ }^{2}$ \\ ${ }^{1}$ Magister en ingeniería, área electrónica, docente investigador de la Universidad Autónoma de \\ Bucaramanga, hgonzalez7@unab.edu.co \\ ${ }^{2}$ Ingeniero Mecatrónico de la Universidad Autónoma de Bucaramanga, yguerrero618@unab.edu.co
}

\begin{abstract}
RESUMEN
El artículo presenta el diseño de un controlador lineal cuadrático gaussiano (LQG) para regular los ángulos de cabeceo, elevación y viaje de un prototipo de un helicóptero de tres grados de libertad (3 DOF). El controlador LQG se diseña a partir de un modelo lineal y se ajustan los parámetros del regulador en base a simulaciones realizadas con el modelo no lineal, evaluando la respuesta transitoria del sistema en lazo cerrado para diferentes valores de la señal de referencia, garantizando obtener la mayor variación de los ángulos alrededor del punto de equilibrio. La validación experimental de la estrategia de control se realiza sobre un prototipo construido por los autores, la respuesta transitoria de los datos simulados se compara con los datos experimentales, para los tres grados de libertad del helicóptero, observando que el modelo matemático se ajusta a la dinámica del prototipo y se cumplen las condiciones de diseño.
\end{abstract}

Palabras clave: Helicóptero 3 DOF, Linealización, Control LQG, Control digital

Recibido: 28 de Septiembre de 2018. Aceptado: 24 de Abril de 2019

Received: September 28, 2018. Accepted: April 24, 2019

DESIGN OF A LQG CONTROLLER FOR A THREE DEGREES OF FREEDOM HELICOPTER

\begin{abstract}
Abstract. This paper presents the design of a linear quadratic gaussian (LQG) controller to regulate the pitch, elevation and travel angles of a helicopter with three degrees of freedom (3 DOF). The LQG controller was designed on the basis of a linear model and the regulator parameters were adjusted based on the simulations performed with the non-linear model, evaluating the transient response of the system in the closed loop for different values of the reference signal, guaranteeing obtain the greatest variation of the angles around the equilibrium point. The experimental validation was done using a prototype built by the authors; the transient response of the simulated data was compared against the experimental data, for the three degrees of freedom of the helicopter, observing that the mathematical model adjusts to the dynamic of the prototype and the conditions of design were fulfilled.
\end{abstract}

Keywords: 3 DOF Helicopter, Linearization, LQG Controller, Digital control

Cómo citar este artículo: H. González, Y. Guerrero, "Diseño de un controlador LQG para un helicóptero de tres grados de libertad", Revista Politécnica, vol. 15, no.28 pp.54-62, 2019. DOI: https://doi.org10.33571/rpolitec.v15n28a5 


\section{INTRODUCCIÓN}

El helicóptero de 3 DOF es un sistema didáctico a escala muy útil e interesante que ha sido utilizado para explicar los efectos aerodinámicos y demostrar en forma práctica diferentes métodos de modelado, análisis y control. El módulo consta de un brazo largo pivotado sobre una base fija vertical, el cual a través de variaciones en el voltaje de dos motores DC, instalados en el extremo del brazo crean una fuerza de empuje, que junto al contrapeso ubicado en el extremo contrario permiten controlar los ángulos de elevación (altura que alcanza el cuerpo del helicóptero), el cabeceo (giro en torno al eje longitudinal del prototipo) y viaje (giro en torno al eje perpendicular al suelo).

Se han establecido diferentes estrategias de control para estabilizar este sistema no lineal. En [1] se diseña un control óptimo basado en la solución de la ecuación de Riccati, SDRE (State Dependents Riccati Equation). En [2] generan la señal de control como la combinación de un compensador por realimentación de espacio de estados y un regulador robusto para estabilizar los ángulos de elevación y cabeceo de un helicóptero 3 DOF. En [3] realizan un trabajo similar, un controlador robusto adaptativo y un regulador lineal cuadrático, esta combinación permite compensar las incertidumbres en los parámetros que presenta el sistema. En [4] se diseña un control en cascada para regular el ángulo de viaje y un lazo independiente para el ángulo de elevación, cada lazo de control presenta un controlador robusto sintonizado mediante la técnica QFT (Quantitative Feedback Theory). En [5] se diseña un control difuso por modo deslizante, esta técnica permite disminuir la presencia de oscilaciones de alta frecuencia en la señal de control. Debido al acoplamiento entre las ecuaciones que definen la dinámica del sistema, en [6] presentan el diseño de un control LQR para cuatro regiones en la cual se observa un comportamiento lineal y por medio de un sistema difuso se elige el controlador óptimo para estabilizar el prototipo en todo el rango de operación. En [7] se presenta el diseño de un control difuso para regular los ángulos de elevación y viaje del helicóptero; las entradas al sistema difuso son el error y la razón de cambio del error, la señal de salida del sistema difuso es integrada para garantizar el punto de consigna. En [8] presentan un control PID adaptativo, es decir, un control difuso ajusta la ganancia proporcional, integral y derivativa en tiempo real del controlador para estabilizar el ángulo de elevación y el de viaje del helicóptero. En [9] presenta el diseño de un controlador no lineal de datos muestreados para un modelo de helicóptero 3 DOF.

El artículo se divide en tres secciones: en la primera se realiza una revisión del estado del arte, en la segunda se presenta el modelo matemático del sistema, en la tercera sección se presenta el diseño del controlador y en la cuarta sección se valida la estrategia de control, comparando los datos experimentales con los datos obtenidos de la simulación, dado una señal de referencia variable para los ángulos de viaje y elevación.

\section{MODELO MATEMÁTICO}

Para determinar el modelo dinámico del helicóptero de tres de libertad (ángulo de elevación, cabeceo y viaje) se aplica el método de Newton Euler [10], dado el diagrama de cuerpo libre de la Fig. 1. Este método establece un equilibrio de fuerzas, analizando los tres puntos de rotación que presenta el helicóptero. Las variables del modelo matemático son el ángulo de elevación $\boldsymbol{\epsilon}$, el ángulo de cabeceo $\boldsymbol{\rho}$, el ángulo de viaje $\tau$ y las fuerzas de empuje de cada uno de los motores, $\boldsymbol{F}_{\boldsymbol{B}}$ y $\boldsymbol{F}_{\boldsymbol{F}}$

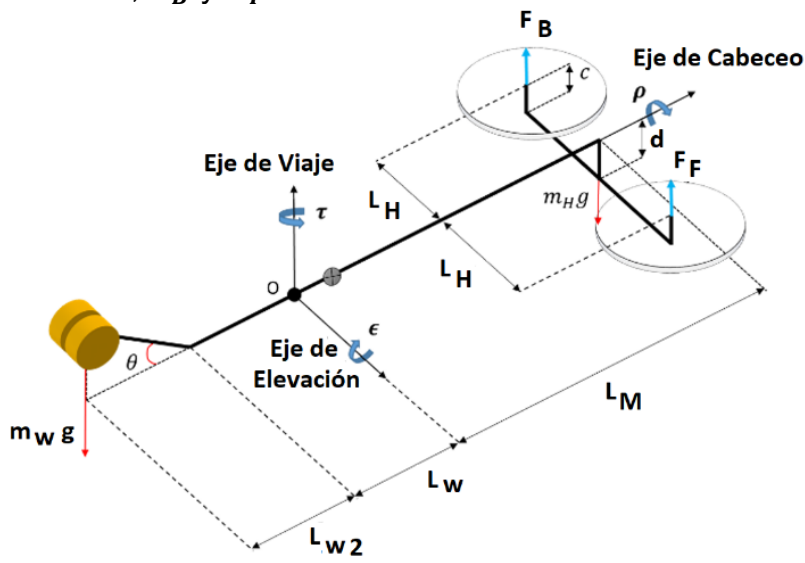

Fig. 1. Representación tridimensional del helicóptero 3 DOF

\subsection{Ecuación para el ángulo de elevación.}

El helicóptero gira respecto al eje perpendicular (ángulo de elevación). Las fuerzas que actúan sobre el sistema es la suma de los empujes provocados por las hélices accionadas por los dos motores DC y los momentos que ejercen el contrapeso y el peso de la viga principal. La ecuación diferencial que rige este movimiento es 


$$
\begin{gathered}
I_{\epsilon} \ddot{\epsilon}=\left(F_{F}+F_{B}\right) L_{M} \cos \rho-\left(I_{\tau}-I_{\rho}\right) \dot{\tau}^{2} \cos \epsilon \sin \epsilon \\
+M_{G}(\epsilon)-k_{d \epsilon} \dot{\epsilon}
\end{gathered}
$$

donde

$$
\begin{array}{r}
M_{G}(\epsilon)=-m_{H} g\left((d-h) \sin \epsilon \cos \rho+L_{M} \cos \epsilon\right)+ \\
m_{W} g\left(L_{W}+L_{W 2} \cos \theta\right) \cos \epsilon+m_{W} g\left(L_{W 2} \sin \theta+\right. \\
h) \sin \epsilon-m_{A} L_{A} g \cos \epsilon
\end{array}
$$

\subsection{Ecuación del ángulo de cabeceo.}

El par principal alrededor del eje de cabeceo se debe a la diferencia de velocidad entre los dos motores, además, cuando $\boldsymbol{\rho} \neq \mathbf{0}$, la fuerza gravitacional del ensamble también producirá un par alrededor del eje de cabeceo. La fricción de la junta y la resistencia del aire se combinan en un única constante $\boldsymbol{k}_{\boldsymbol{d} \rho}$. La ecuación diferencial que describe este movimiento es:

$$
I_{\rho} \ddot{\rho}=\left(F_{B}-F_{F}\right) L_{H}-m_{H} g(d-c) \sin \rho \cos \epsilon-k_{d \rho} \dot{\rho}
$$

\subsection{Ecuación del ángulo de viaje.}

Las fuerzas que actúan sobre el eje de viaje se deben a la diferencia entre los empujes de los dos motores. La ecuación diferencial es

$$
I_{\tau}(\epsilon) \ddot{\tau}=\left(F_{F}+F_{B}\right) L_{M} \cos \epsilon \sin \rho-k_{d \tau} \dot{\tau}
$$

donde

$$
I_{\tau}(\epsilon)=(\cos \epsilon)^{2}\left(m_{W}\left(L_{W}+L_{W 2} \cos \theta\right)^{2}+m_{H} L_{M}^{2}\right)
$$

\section{$2.4 \quad$ Modelo lineal}

A partir de las ecuaciones (1), (3) y (4), que gobiernan la dinámica del sistema, se determina un modelo lineal, para lo cual se aproxima los términos $\sin x \cong x$ y $\cos x \cong 1$, donde $x$ denota el ángulo a linealizar (elevación, cabeceo o viaje). El término $\dot{\boldsymbol{t}}^{2}$, razón de cambio del ángulo de viaje elevado al cuadrado, de la ecuación (1), se desprecia en la etapa de linealización porque al ser variaciones pequeñas este valor tiende a cero. Al realizar estas aproximaciones se obtienen las ecuaciones lineales (6), (7) y (8) para los tres ángulos, donde $\boldsymbol{F}_{\text {sum }}=$ $\boldsymbol{F}_{\boldsymbol{B}}+\boldsymbol{F}_{\boldsymbol{F}}$ y $\boldsymbol{F}_{\text {dif }}=\boldsymbol{F}_{\boldsymbol{B}}-\boldsymbol{F}_{\boldsymbol{F}}$. La variable $\boldsymbol{F}_{\text {sum }}$ corresponde a la fuerza de empuje que permite elevar el helicóptero y $\boldsymbol{F}_{\text {dif }}$ la fuerza de empuje que genera una variación del ángulo de cabeceo. En la tabla 1 se presenta el valor de los parámetros del modelo matemático. Estos valores corresponden al prototipo que se diseñó para validar la estrategia de control, el cual se describe en la sección 4.

$$
\begin{aligned}
& I_{\epsilon} \ddot{\epsilon}=F_{\text {sum }} L_{M}-m_{H} g\left((d-h) \epsilon+L_{M}\right) \\
& +m_{W} g\left(L_{W}+L_{W 2} \cos \theta\right) \\
& +m_{W} g\left(L_{W 2} \sin \theta+h\right) \epsilon \\
& \text { - } m_{A} g L_{A}-k_{d \epsilon} \dot{\epsilon} \\
& I_{\rho} \ddot{\rho}=F_{d i f} L_{H}-m_{H} g(d-c) \rho-k_{d \rho} \dot{\rho} \\
& I_{\tau}(0) \ddot{\tau}=F_{\text {sum }} L_{M} \rho-k_{d \tau} \dot{\tau} \\
& I_{\tau}(0)=\left(m_{W}\left(L_{W}+L_{W 2} \cos \theta\right)^{2}+m_{H} L_{M}{ }^{2}\right)
\end{aligned}
$$

Asumiendo como vector de estados $x(t)=$ [ $\boldsymbol{\rho}(\boldsymbol{t}) ; \dot{\boldsymbol{\rho}}(\boldsymbol{t}) ; \boldsymbol{\epsilon}(\boldsymbol{t}) ; \dot{\boldsymbol{\epsilon}}(\boldsymbol{t}) ; \boldsymbol{\tau}(\boldsymbol{t}) ; \dot{\boldsymbol{\tau}}(\boldsymbol{t})]$, vector de entrada $\boldsymbol{u}(\boldsymbol{t})=\left[\boldsymbol{F}_{\text {sum }}(\boldsymbol{t}) ; \boldsymbol{F}_{\text {dif }}(\boldsymbol{t})\right]$ y los siguientes puntos de equilibrio $x_{10}=x_{20}=x_{30}=x_{40}=x_{50}=x_{60}=0$, el modelo lineal en espacio de estados [11] se define por las siguientes ecuaciones.

$$
\begin{gathered}
\dot{x}(t)=A_{L} x(t)+B_{L} u(t) \\
y(t)=C_{L} x(t)
\end{gathered}
$$

$$
\begin{gathered}
A_{L}=\left[\begin{array}{cccccc}
0 & 1 & 0 & 0 & 0 & 0 \\
-\frac{m_{H} g(c-d)}{I_{\rho}} & -\frac{k_{d \rho}}{I_{\rho}} & 0 & 0 & 0 & 0 \\
0 & 0 & 0 & 1 & 0 & 0 \\
0 & 0 & \frac{N}{I_{\epsilon}} & -\frac{k_{d \epsilon}}{I_{\epsilon}} & 0 & 0 \\
0 & 0 & 0 & 0 & 0 & 1 \\
-\frac{L_{M} F_{\text {sum }}^{0}}{I_{\tau \epsilon}} & 0 & 0 & 0 & 0 & -\frac{k_{d \tau}}{I_{\tau \epsilon}}
\end{array}\right] \\
N=\left(m_{W} g\left(h+L_{W 2} \sin (\theta)\right)-m_{H} g(d-h)\right) \\
F_{\text {sum }}^{0}=\frac{L_{A} g m_{A}-g m_{W}\left(L_{W}+L_{W 2} \cos (\theta)\right)+L_{M} g m_{H}}{L_{M}}
\end{gathered}
$$

$$
\begin{gathered}
B_{L}=\left[\begin{array}{cc}
0 & 0 \\
0 & \frac{L_{H}}{I_{\rho}} \\
0 & 0 \\
\frac{L_{M}}{I_{\epsilon}} & 0 \\
0 & 0 \\
0 & 0
\end{array}\right] \\
C_{L}=\left[\begin{array}{llllll}
0 & 0 & 1 & 0 & 0 & 0 \\
0 & 0 & 0 & 0 & 1 & 0 \\
1 & 0 & 0 & 0 & 0 & 0
\end{array}\right]
\end{gathered}
$$


Tabla 1. Valor de los parámetros del modelo matemático de un helicóptero 3 DOF

\begin{tabular}{|c|c|}
\hline Parámetro & Valor \\
\hline Masa del helicóptero $m_{H}$ & $1.160 \mathrm{~kg}$ \\
\hline Masa del contrapeso $m_{W}$ & $1.015 \mathrm{~kg}$ \\
\hline Masa de la viga principal $m_{A}$ & $0.19367 \mathrm{~kg}$ \\
\hline $\begin{array}{l}\text { Distancia entre el eje de elevación y el } \\
\text { pliegue del contrapeso } L_{W}\end{array}$ & $0.47 \mathrm{~m}$ \\
\hline $\begin{array}{l}\text { Distancia entre el pliegue del } \\
\text { contrapeso hasta le contrapeso } L_{W 2}\end{array}$ & $0.10762 \mathrm{~m}$ \\
\hline $\begin{array}{l}\text { Distancia entre el eje de elevación y el } \\
\text { helicóptero } L_{M}\end{array}$ & $0.5775 \mathrm{~m}$ \\
\hline $\begin{array}{l}\text { Distancia entre el eje de cabeceo y el } \\
\text { centro de las hélices } L_{H}\end{array}$ & $0.19 \mathrm{~m}$ \\
\hline $\begin{array}{l}\text { Distancia entre el eje de elevación y el } \\
\text { centro de masa de la viga principal } L_{A}\end{array}$ & $0.10565 \mathrm{~m}$ \\
\hline $\begin{array}{l}\text { Distancia entre el eje de cabeceo y la } \\
\text { base de los motores } d\end{array}$ & $0.125 \mathrm{~m}$ \\
\hline $\begin{array}{l}\text { Distancia entre la base de los motores y } \\
\text { las hélices } c\end{array}$ & $0.05269 \mathrm{~m}$ \\
\hline $\begin{array}{l}\text { Distancia entre eje de elevación y el } \\
\text { centro de la viga principal } h\end{array}$ & $0.01862 \mathrm{~m}$ \\
\hline $\begin{array}{l}\text { Inercia de rotación alrededor del eje de } \\
\text { cabeceo } I_{p}\end{array}$ & $\begin{array}{c}0.04794 \\
\mathrm{kgm}^{2}\end{array}$ \\
\hline $\begin{array}{l}\text { La inercia de rotación alrededor del eje } \\
\text { de elevación } I_{\epsilon}\end{array}$ & $\begin{array}{c}0.64562 \\
\mathrm{kgm}^{2}\end{array}$ \\
\hline $\begin{array}{l}\text { Coeficiente de fricción dinámico eje de } \\
\text { cabeceo } k_{d p}\end{array}$ & $\begin{array}{c}0.053 \\
\mathrm{Nms} / \mathrm{rad}\end{array}$ \\
\hline $\begin{array}{l}\text { Coeficiente de fricción dinámico eje de } \\
\text { elevación } k_{d \epsilon}\end{array}$ & $1 \mathrm{Nms} / \mathrm{rad}$ \\
\hline $\begin{array}{l}\text { Coeficiente de fricción dinámico eje de } \\
\text { viaje } k_{d \tau}\end{array}$ & $\begin{array}{l}0.5 \mathrm{Nms} / \\
\mathrm{rad}\end{array}$ \\
\hline
\end{tabular}

\section{SISTEMA DE CONTROL POR ESPACIO DE ESTADOS}

El principio básico del diseño de un sistema de control por espacio de estados es determinar dos matrices de ganancias, $K$ y $K_{i}$, la primera que multiplique a los estados del sistema y la segunda al integrador ubicado en el camino directo entre el comparador de error y la planta [12], tal como se observa en la figura 2. Asumiendo que la entrada de referencia (función escalón) se aplica en $t=0$, la dinámica del sistema para $t>0$ esta descrita por las siguientes ecuaciones:

$$
\begin{gathered}
{\left[\begin{array}{c}
x(k+1) \\
v(k+1)
\end{array}\right]=\left[\begin{array}{cc}
G_{L} & 0 \\
-C_{L} G_{L} & I
\end{array}\right]\left[\begin{array}{l}
x(k) \\
v(k)
\end{array}\right]+\left[\begin{array}{c}
H_{L} \\
-C_{L} H_{L}
\end{array}\right] u(k)} \\
+\left[\begin{array}{l}
0 \\
I
\end{array}\right] r(k+1)
\end{gathered}
$$

donde $G_{L}$ es la matriz de estados, representada en tiempo discreto, la cual se determina a partir de la matriz exponencial, $G_{L}=e^{A_{L} T_{m}}\left(T_{m}\right.$ corresponde al periodo de muestreo), y $H_{L}$ es la matriz de entrada, representada en tiempo discreto, que se calcula a partir de la relación $H_{L}=A_{L}^{-1}\left(G_{L}-I\right) B_{L}$.

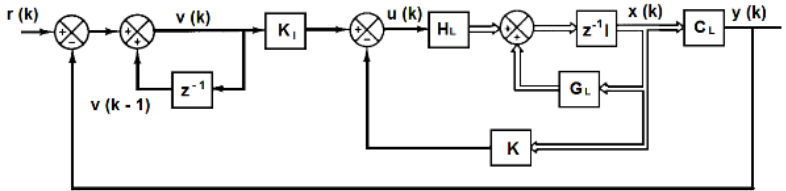

Fig. 2. Diagrama de bloques del sistema de seguimiento

Considerando que $r(k)$ es una entrada escalón, se tiene que $r(k)=r(k+1)=r$ (constante). Cuando $k$ tiende a infinito, la ecuación de estados se define como:

$$
\begin{gathered}
{\left[\begin{array}{l}
x(\infty) \\
v(\infty)
\end{array}\right]=\left[\begin{array}{cc}
G_{L} & 0 \\
-C_{L} G_{L} & I
\end{array}\right]\left[\begin{array}{l}
x(\infty) \\
v(\infty)
\end{array}\right]+\left[\begin{array}{c}
H_{L} \\
-C_{L} H_{L}
\end{array}\right] u(\infty)} \\
+\left[\begin{array}{l}
0 \\
I
\end{array}\right] r(\infty)
\end{gathered}
$$

Restando la ecuación (14) de la ecuación (15), se obtiene:

$$
\begin{aligned}
{\left[\begin{array}{c}
x_{e}(k+1) \\
v_{e}(k+1)
\end{array}\right] } & =\left[\begin{array}{cc}
G_{L} & 0 \\
-C_{L} G_{L} & 1
\end{array}\right]\left[\begin{array}{l}
x_{e}(k) \\
v_{e}(k)
\end{array}\right] \\
& +\left[\begin{array}{c}
H_{L} \\
-C_{L} H_{L}
\end{array}\right] u_{e}(k)
\end{aligned}
$$

donde

$$
\begin{gathered}
x_{e}(k)=x(k)-x(\infty) \\
v_{e}(k)=v(k)-v(\infty) \\
u_{e}(k)=-\boldsymbol{K} x_{e}(k)+\boldsymbol{K}_{I} v_{e}(k)
\end{gathered}
$$

Definiendo un nuevo vector de estados, $\xi(k)=$ $\left[x_{e}(k) ; v_{e}(k)\right]$, la dinámica del sistema en lazo cerrado se representa:

$$
\begin{gathered}
\xi(k+1)=\widehat{\boldsymbol{G}} \xi(k)+\widehat{\boldsymbol{H}} u_{e}(k) \\
u_{e}(k)=\widehat{\boldsymbol{K}} \xi(k)
\end{gathered}
$$

donde,

$$
\begin{aligned}
& \widehat{G}=\left[\begin{array}{cc}
G_{L} & 0 \\
-C_{L} G_{L} & 1
\end{array}\right] \quad \widehat{H}=\left[\begin{array}{c}
H_{L} \\
-C_{L} H_{L}
\end{array}\right] \\
& \widehat{K}=\left[\begin{array}{ll}
K & -K_{I}
\end{array}\right]
\end{aligned}
$$




\subsection{Control LQG (Linear Quadratic Gaussian Control)}

El controlador LQG es la combinación de una ganancia de realimentación de estados y un estimador de estados, tipo Kalman [13]. El primer paso para el diseño del controlador LQG es determinar una matriz de ganancias $\widehat{K}$ que minimice la función de costo $J$, ecuación (23). Las matrices $Q$ y $R$ son matrices definidas positivas, generalmente diagonales, que determinan el equilibrio entre el seguimiento de una trayectoria por parte de los estados y la energía de la señal de control requerida por el sistema para alcanzar los objetivos de control deseados. La matriz $\widehat{K}$ se determina resolviendo la ecuación matricial de Riccatti, dadas las matrices $\widehat{G}$, $\widehat{H}, Q$ y $R$.

$$
J=\frac{1}{2} \sum_{k=0}^{\infty}\left(\xi^{T} Q \xi+u_{e}^{T} R u_{e}\right)
$$

Para estimar los estados se utiliza un filtro Kalman, el cual asume la presencia de ruido gaussiano en la señal de control y la variable a medir en el proceso. Conocidas las varianzas del ruido de estas dos señales, $Q_{n}$ y $R_{n}$, se determina un vector de ganancias $L$ que minimiza el error en la estimación, $x-\hat{x}$, utilizando la ecuación de Riccatti. Matemáticamente, los estados se calculan a partir de la siguiente expresión:

$$
\hat{x}(k+1)=G_{L} \hat{x}(k)+H_{L} u(k)+L\left(y(k)-C_{L} \hat{x}(k)\right)
$$

donde $u(k)$ es la señal de control y $y(k)$ la variable a controlar del proceso. Para el diseño del sistema de control LQG, en primer lugar, se discretiza la representación en espacio de estados (Ec. 10) con un periodo de muestreo de $T_{m}=0.01 \mathrm{sg}$, el cual se determinó a partir del criterio de Nyquist, estableciendo un ancho de banda de sistema de $20 \mathrm{~Hz}$. El siguiente paso es definir las matrices diagonales $Q$ y $R$

$$
\begin{gathered}
Q=\operatorname{diag}[1,1,1,1,20,200,0.6,1] \\
R=\operatorname{diag}[(10,1000)]
\end{gathered}
$$

Se soluciona la ecuación de Riccatti, la cual permite determinar la matriz $\widehat{K}$ que minimiza la función de optimización establecida en la ecuación (23), conocidas las matrices $\widehat{G}$ y $\widehat{H}$, ecuación (22).

$$
\begin{aligned}
& \widehat{K}=\left[\begin{array}{ccc}
0 & 0 & 17.1789 \\
0.8238 & 0.4243 & 0
\end{array} \ldots\right. \\
& \begin{array}{cccc}
4.7262 & 0 & 0 & \\
0 & 6.2361 & 4.2024 & \cdots . .
\end{array} \\
& \left.\begin{array}{cc}
-0.2398 & -0.0039 \\
-0.000002 & -0.0313
\end{array}\right]
\end{aligned}
$$

Para el diseño del observador se determina la varianza presente en la señal del actuador y el sensor, para lo cual se fija una fuerza de empuje constante que garantice mantener el sistema en equilibrio, se registra las fuerzas $F_{\text {sum }}$ y $F_{\text {dif }}$, además los ángulos de elevación y viaje. Se determina la varianza de estas cuatro señales para generar las matrices $Q_{n}$ y $R_{n}$, respectivamente, y se soluciona la ecuación de Ricatti para determinar la matriz $L$.

$$
\begin{gathered}
Q_{n}=\operatorname{diag}\left[0.015^{2}, 0.03^{2}\right] \\
R_{n}=\operatorname{diag}\left[0.25^{2}, 0.01^{2}\right] \\
L=\left[\begin{array}{cc}
-0.003 & 2.105891 \\
0.004 & -1.6549 \\
0.088 & 0.050 \\
0.3763 & 0.0215 \\
0 & 0.1442 \\
-0.001 & 1.001
\end{array}\right]
\end{gathered}
$$

Para evaluar la respuesta transitoria del sistema en lazo cerrado se implementa en la herramienta de simulink el modelo no lineal con el regulador LQG, el cual incluye el controlador y el observador. En la figura 3 se presenta el diagrama de bloques que se implementó, el bloque de color rojo representa el modelo no lineal, con un cuadrado de color rojo se demarca el controlador y con un cuadro de color celeste el observador. En la simulación se adiciona el ruido del sensor y el actuador definidos por las matrices $Q_{n}$ y $R_{n}$. Se evalúa la respuesta transitoria del sistema en lazo cerrado ante una entrada escalón, logrando un tiempo de establecimiento de $3.32 s$ y $4.63 s$ para el ángulo de elevación y viaje, respectivamente, y un sobrepaso inferior a $6.53 \%$ para ambas señales.

Con el objetivo de analizar el rango de variación que se puede obtener para los ángulos de elevación y 
viaje alrededor del punto de equilibrio se realizan simulaciones modificando de forma aleatoria la señal de referencia. En la figura 4 se presentan los resultados, se observa que variaciones en un rango de $30^{\circ}$ en el ángulo de elevación, la respuesta del modelo lineal y no lineal se aproximan, con un incremento en el sobrepaso en los datos relacionados con el modelo no lineal, manteniéndose cercanos el tiempo de levantamiento y el tiempo de establecimiento. En el ángulo de viaje se presenta el mismo comportamiento con una variación de $90^{\circ}$.

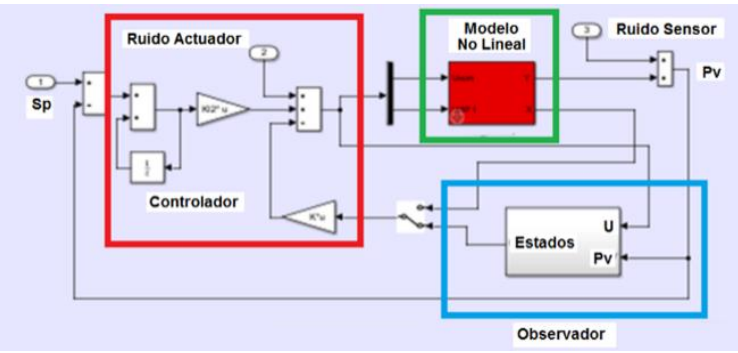

Fig. 3. Diagrama de bloques del sistema en lazo cerrado: controlador LQG y modelo no lineal del helicóptero 3 DOF.

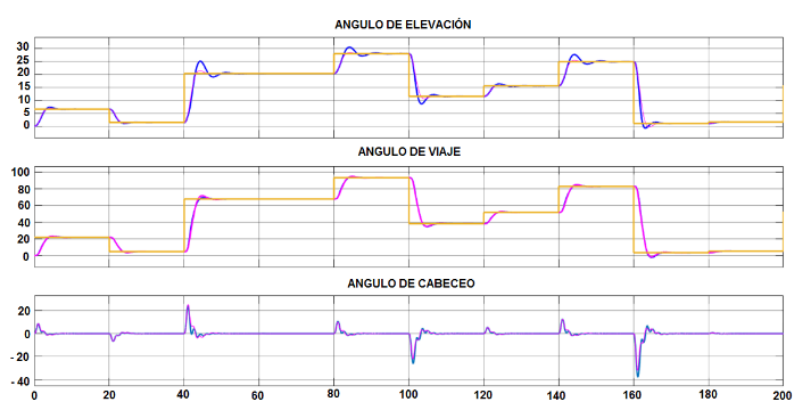

Fig. 4. Respuesta transitoria de los ángulos de elevación, viaje y cabeceo de un helicóptero 3 DOF dado el modelo lineal y no lineal

\section{VALIDACIÓN EXPERIMENTAL}

Con el objetivo de validar la estrategia de control se diseñó un prototipo de un helicóptero de 3 DOF. EI dispositivo presenta un rango de 65 grados para el ángulo de elevación, +41 por encima de la horizontal y -24 grados por debajo; 60 grados para el ángulo de cabeceo ( \pm 30 grados) y 360 grados para el ángulo de viaje ( \pm 180 grados). El ángulo de elevación y cabeceo se miden por medio de un potenciómetro lineal, el ángulo de viaje se registra por medio de un encoder absoluto de 1024 pulsos por revolución. Los motores que generan el empuje para mover el prototipo son marca Pittman de 24 voltios DC (Fig. 5). La estrategia de control se implementa en la herramienta de Simulink de Matlab y se establece una comunicación entre este software y Arduino Due, el cual hace las funciones de tarjeta de adquisición y generador de las señales de control para los motores DC [14] [15].

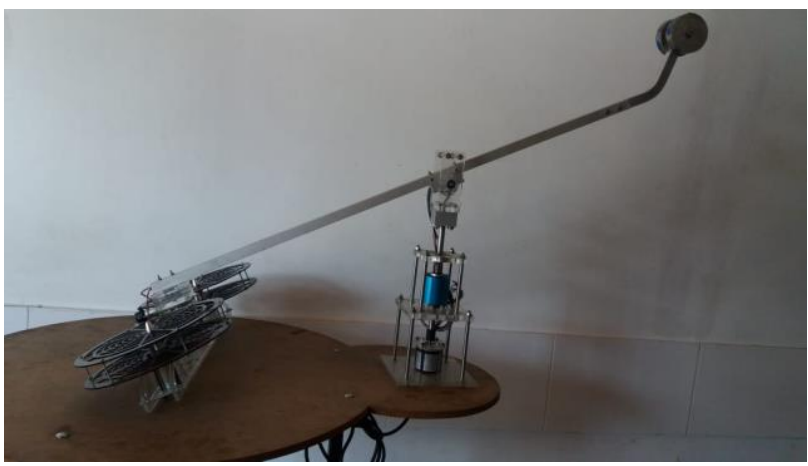

Fig. 5. Prototipo de Helicóptero de 3 DOF

Se evalúa la respuesta transitoria del sistema en lazo cerrado, asignando diferentes valores para el ángulo de elevación y el ángulo de viaje. En la figura 6 se presenta la comparación entre los datos experimentales (línea azul) y los datos simulados (línea roja), dada una señal de referencia variable (línea verde), para el ángulo de elevación y el ángulo de viaje. El primer ángulo varía en un rango de $\pm 25^{\circ}$ y el segundo varía de $-50^{\circ}$ a $100^{\circ}$. El modelo matemático se ajusta a la dinámica del sistema, el filtro de Kalman estima de forma correcta el valor de los estados, esta característica se logra porque en la etapa de diseño se considera el ruido presente en las señales de control y los sensores, además se garantiza que los polos del observador se encuentren detrás de los polos del controlador. Al realizar el prototipo un giro sobre su propio eje debe modificar el ángulo de cabeceo, una vez el ángulo de viaje se acerca a la referencia establecida, su valor se debe acercar a cero, pero esta relación no se mantiene porque los dos motores no presentan la misma curva de voltaje versus fuerza de empuje. El ángulo de elevación presenta un sobrepaso mayor cuando va descendiendo, lo cual se debe al efecto 
de la gravedad. Para minimizar este fenómeno se han establecido técnicas de control no lineal que permiten a la vez compensar las incertidumbres en los parámetros del modelo. Mohit Mehndiratta, et al, realiza una comparación entre una técnica lineal y una estrategia no lineal, para regular los ángulos de elevación y viaje del prototipo de helicóptero 3 DOF de Quanser [16]. Inicialmente sintoniza un controlador PID basado en la técnica de LQR, esta estrategia genera sobrepasos de magnitud considerable (Fig. 7) ante una entrada en forma de onda cuadrada y no responde de forma adecuada a una perturbación que simula el comportamiento del viento. Para mejora la respuesta transitoria sintoniza un controlador difuso SIT2-FPID, técnica que se fundamenta en ubicar un sistema difuso entre la señal del error y la entrada al controlador PID.
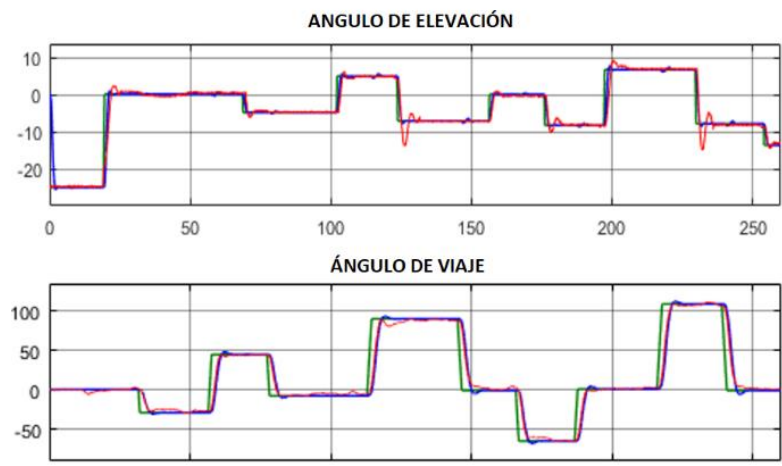

ÁNGULO DE CABECEO

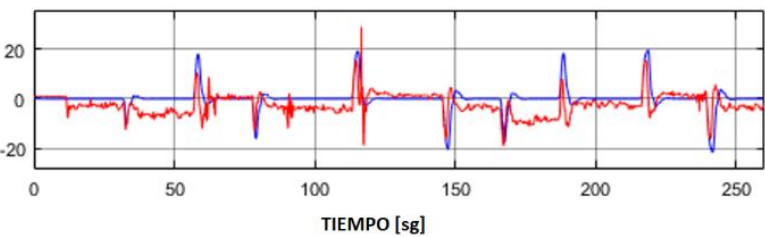

Fig. 6. Respuesta transitoria del controlador LQG Línea de color verde corresponde a la señal de referencia, la línea de color azul a los datos experimentales y la línea de color roja a los datos simulados. a) Ángulo de elevación, b) ángulo de viaje, c) ángulo de cabeceo
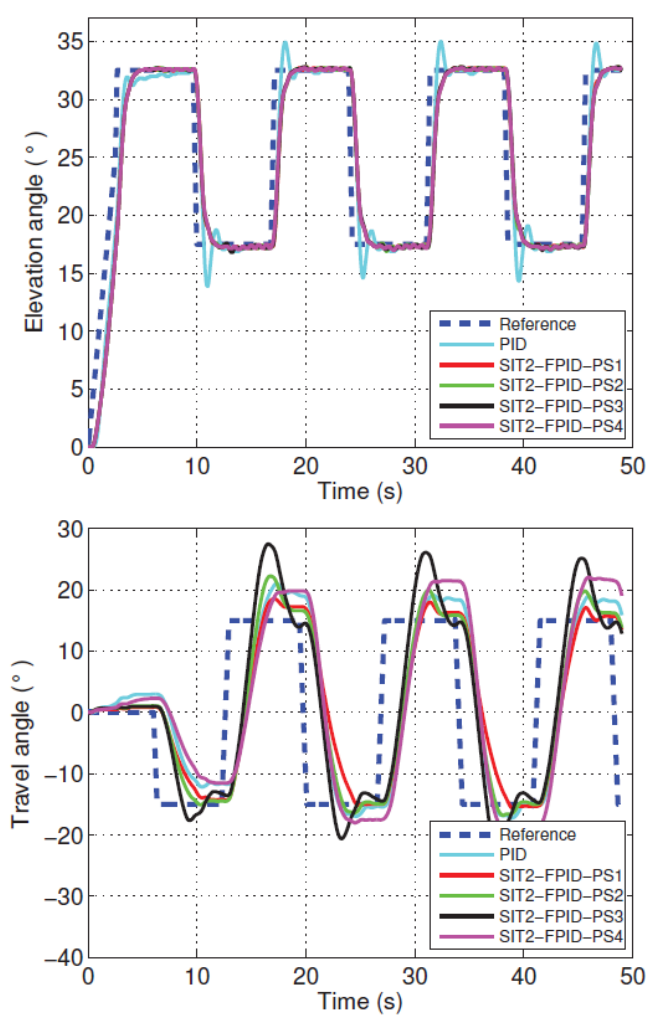

Fig. 7. Respuesta transitoria de un controlador difuso SIT2-FPID. La imagen es tomada del artículo Design and Experimental Validation of Single Input Type-2 Fuzzy PID Controllers as Applied to 3 DOF Helicopter Testbed [16]

\section{CONCLUSIONES}

El helicóptero de tres grados de libertad es una herramienta académica que permite validar diferentes estrategias de control lineal y no lineal. En el artículo se presenta el modelo matemático y el diseño de un control LQG para este prototipo. La técnica lineal LQG permite un buen seguimiento de la señal de referencia y el filtro de Kalman estima de forma rápida y precisa la información de los estados, logrando estabilizar los ángulos de elevación, cabeceo y viaje. Técnicas lineales se puede aplicar para esta clase de prototipos, pero se observa que no compensan de forma adecuada fuerzas gravitatorias, por lo cual se recomienda la implementación de técnicas de control no lineal.

La integración de Simulink con Arduino permite la validación de estrategias de control avanzado, reduciendo el tiempo para programar el regulador, 
porque se puede realizar a partir de los bloques de Simulink, sin necesidad de escribir el código. El problema que presenta esta modalidad es que no se garantizar una operación en tiempo real cuando el periodo de muestreo es inferior a $0.01 \mathrm{~s}$.

\section{AGRADECIMIENTOS}

Los autores agradecen a la Universidad Autónoma de Bucaramanga por facilitar los recursos económicos para la construcción del prototipo de un helicóptero de tres grados de libertad.

\section{REFERENCIAS BIBLIOGRÁFICAS}

[1] Ahmet Çağrı Arıcan; Sinan Ozcan; Bedrettin Mahmut Kocagil; Ümit Müfit Güzey; Metin U. Salamci. "Suboptimal control of a 3 dof helicopter with state dependent riccati equations". XXVI International Conference on Communication and Automation Technologies (ICAT), 2017. DOI: 10.1109/ICAT.2017.8171615

[2] Liu Hao, Yu Yao, Lu Geng, Zhong Yisheng. "Robust LQR attitude control of 3 DOF helicopter". Proceedings of the 29th Chinese Control Conference. July 29-31, 2010, Beijing, China. DOI: 10.1109/TIE.2012.2216233

[3] R. I. Boby, H. Mansor, T. S. Gunawan, S. Khan. "Robust Adaptive LQR Control of Nonlinear System Application to 3-Dof Flight Control System". Proceedings of the IEEE International Conference on Smart Instrumentation, Measurement and Applications. DOI: 10.1109/ICSIMA.2014.7047443

[4] Ameerul Hakeem Mohd Hairon, Hasmah Mansor, Teddy Surya Gunawan, Sheroz Khan. "Robust Control of Bench-top Helicopter Using Quantitative Feedback Theory". TELKOMNIKA Indonesian Journal of Electrical Engineering, pp 500 - 507. 2015. DOI: 10.11591/telkomnika.v14i3.7899

[5] Lei Yang, Lidong Zhang, Qing Li. "Design and application of fuzzy sliding mode control in the 3DOF helicopter". International Workshop on Intelligent Systems and Applications, ISA 2009. DOI: 10.1109/IWISA.2009.5072806.

[6] Zhichao Liu, Zouhair Choukri, Hongbo Shi. "Control strategy design base on fuzzy logic and LQR for 3 DOF helicopter model". International Conference on Intelligent Control and Information
Processing, ICICIP 2010. DOI: 10.1109/ICICIP.2010.5564250

[7] Kamil Karaman, Yusuf Talha Bekaroğlu, Mehmet Turan Söylemez, Kemal Uçak, Gülay Öke Günel. "Control 3 DOF helicopter via fuzzy PID controller". 9th International Conference on Electrical and Electronics Engineering, ELECO 2015. DOI: 10.1109/ELECO.2015.7394494

[8] González Hernando, Arizmendi Carlos, García Joan, Angulo Alexander, Herrera Cristian. "Design and Experimental Validation of Adaptive Fuzzy PID Controller for a Three Degrees of Freedom Helicopter". 2018 IEEE International Conference on Fuzzy Systems (FUZZ-IEEE). ISBN 978-1-50906020-7. 2018

[9] K. Takamuku, M. Ishitobi ${ }^{*}$, T. Kumada, M. Nishi and S. Kunimatsu. "Redesign Implementation of a Nonlinear Sampled-Data Controller for a 3-DOF Model Helicopter". SICE Annual Conference 2011.

[10] Jerry Ginsberg. "Engineering Dynamics". Cambridge University Press. 2008. ISBN:13: 9780521883030

[11] Katsuhiko Ogata. "Ingeniería de Control Moderna”. Quinta Edición. Pearson Educación, 2010. ISBN: 9788483226605

[12] Katsuhiko Ogata. "Sistemas de control en tiempo discreto". Segunda Edición. Pearson Educación, 1995. ISBN: 9688805394

[13] Roland S. Burns. "Advanced Control Engineering". Butterworth-Heinemann Books. ISBN 978-0-7506-5100-4. 2001. DOI 10.1016/B978-07506-5100-4.X5000-1

[14] Shamsul Aizam Zulkifli, Mohd Najib Hussin, Abdul Salam Saad. "MATLAB-Arduino as a low cost microcontroller for 3 phase inverter". IEEE Student Conference on Research and Development (SCOReD), 2014.

[15] González L. Juan Carlos, León S. Gabriel, Huamani N. Pedro. "Designing of control using Matlab and Arduino for a electrohydraulic system from seismic simulation". IEEE XXIV International Conference on Electronics, Electrical Engineering and Computing (INTERCON), 2017 
[16] Mohit Mehndiratta, Erdal Kayacan, Tufan Kumbasar. "Design and Experimental Validation of Single Input Type-2 Fuzzy PID Controllers as Applied to 3 DOF Helicopter Testbed". IEEE International Conference on Fuzzy Systems (FUZZIEEE), 2016. DOI: 10.1109/FUZZIEEE.2016.7737879 